\title{
Calibration of radii and masses of open clusters with a simulation
}

\author{
A. Ernst ${ }^{1}$, A. Just ${ }^{1}$, P. Berczik ${ }^{1,2,3}$, and M. I. Petrov ${ }^{4}$ \\ 1 Astronomisches Rechen-Institut, Zentrum für Astronomie der Universität Heidelberg, Mönchhofstrasse 12-14, 69120 Heidelberg, \\ Germany \\ e-mail: aernst@ari.uni-heidelberg.de \\ 2 National Astronomical Observatories of China, Chinese Academy of Sciences, Datun Lu 20A, Chaoyang District, Beijing 100012 , \\ PR China \\ 3 Main Astronomical Observatory, National Academy of Sciences of Ukraine, Akademika Zabolotnoho 27, 03680 Kyiv, Ukraine \\ ${ }^{4}$ Institut für Astronomie der Universität Wien, Türkenschanzstraße 17, 1180 Wien, Austria \\ Received 30 April 2010 / Accepted 1 September 2010
}

\section{ABSTRACT}

\begin{abstract}
Context. Piskunov and collaborators developed a method to make a simple mass estimate for tidally limited star clusters based on the identification of the tidal radius in a King profile with the dynamical Jacobi radius. The application of this method to an unbiased open cluster catalog yielded significantly higher cluster masses than the classical methods.

Aims. We quantify the bias in the mass determination as a function of projection direction and cluster age by analyzing a simulated star cluster.

Methods. We use direct $N$-body simulations of a star cluster in an analytic Milky Way potential that account for stellar evolution and apply a best fit to the projected number density of cluster stars.

Results. We obtain significantly overestimated star cluster masses that depend strongly on the viewing direction. The overestimation is typically in the range of $10-50$ percent and reaches a factor of 3.5 for young clusters. Mass segregation reduces the derived limiting radii systematically.
\end{abstract}

Key words. methods: statistical - methods: data analaysis - open clusters and associations: general - solar neighborhood

\section{Introduction}

Piskunov et al. (2007, 2008a,b) developed an approach to determining the masses of open star clusters (OCs) and applied it to determine the initial and present-day mass function of OCs in the solar neighborhood. The method is based on the determination of the tidal radius $r_{\mathrm{t}}$ from the cumulative number of cluster members as a function of projected distance from the cluster center. For each cluster, the tidal radius $r_{\mathrm{t}}$ is determined from projected number density profiles by fitting a King 1962 profile (King 1962). The identification of the King cutoff radius $r_{\mathrm{t}}$ with the "Jacobi" radius $r_{\mathrm{J}}$ (i.e., the dynamical tidal radius, which is the distance from the cluster center to the Lagrange points $L_{1}$ and $L_{2}$ ) then yields the OC mass from the standard formula given here by Eq. (9) solved for $M_{\mathrm{cl}}$. The application of this dynamical mass estimate for tidally limited clusters to an unbiased OC catalog yields a mass determination that is independent of the classical methods. A detailed comparison with other cluster mass determinations is also made. In a second step, the method is extended to all OCs of an unbiased cluster catalog by establishing a transformation of the observed semi-major axis and central surface density to $r_{\mathrm{t}}$. These results were then used to derive the cluster present-day mass function (CPDMF) and the initial mass function of OCs (CIMF) in the extended solar neighborhood. Adopting a constant cluster formation rate over the past $10 \mathrm{Gyr}$ yields a surface density of $18 M_{\odot} \mathrm{pc}^{-2}$ of stars born in OCs. This corresponds to a fraction of $37 \%$ of disk stars born in OCs (Röser et al. 2010). This is large compared to the classical values of the order of 10\% or less (e.g., Wielen 1971; Miller \& Scalo 1978).

Some crucial assumptions enter the dynamical mass determination based on fitting a King profile: a) The OC fills its Roche lobe in the tidal field of the Milky Way. For compact (e.g.
Roche-lobe underfilling) clusters, $r_{\mathrm{J}}$ and as a consequence the mass can be underestimated by a large amount; b) The effect of mass segregation can be neglected, i.e., star counts on the upper main sequence, which dominate the observed cluster members, are representative of the mass distribution; c) The elliptic shape of the clusters and the contamination by tidal tail stars do not result in a systematic bias. Shape parameters were measured by Kharchenko et al. (2009) and the distribution of tidal tail stars have been investigated in detail (e.g. Just et al. 2009); d) The tidal radius $r_{\mathrm{t}}$ determined by fitting the cumulative projected mass profile represents the Jacobi radius $r_{\mathrm{J}}$ used to derive the cluster mass. Since the cluster mass depends on the third power of $r_{\mathrm{J}}$, the method is very sensitive to systematic errors in the derivation of $r_{\mathrm{J}}$.

In the present paper, we quantify the possible bias introduced by identifying the tidal radius $r_{\mathrm{t}}$ inferred from a King profile fitting with the Jacobi radius $r_{\mathrm{J}}$ used for the mass determination by Piskunov et al. (2007). We applied the King profile fitting procedure to a direct $N$-body simulation of a dissolving star cluster at different evolutionary states. We simulated a star cluster on a circular orbit at $R_{\mathrm{C}}=8.5 \mathrm{kpc}$ which evolved in the tidal field of the Milky Way including stellar evolution. We took snapshots of the evolved model with all stellar masses and positions at four different times and projected the snapshots from the perspective of an observer on Earth (at $R_{0}=8 \mathrm{kpc}$ ) onto the sky, at different positions along its orbit. After all, we determined the model's limiting radius $r_{\mathrm{t}}$ by fitting the projected cumulative mass profile with Eq. (6) and compared $r_{\mathrm{t}}$ to the actual Jacobi radius $r_{\mathrm{J}}$.

The paper is organized as follows. In Sect. 2 we discuss the method of $\mathrm{N}$-body simulations in the external potential of the Milky Way and Sect. 3 contains the theory of the cluster 
geometry in a tidal field. In Sect. 4, we show a simple iterative method to determine the Jacobi radius of an $N$-body model of a star cluster in a tidal field. In Sect. 5, the projection and fitting methods are described. Finally, Sect. 6 contains our results and Sect. 7 our conclusions.

\section{Numerical simulation}

We analyze in detail a numerical simulation of a star cluster with initial mass $M_{0}=10^{4} M_{\odot}$ on a circular orbit in an analytic Milky Way potential. It is the fiducial cluster simulation (run 10) discussed in Just et al. (2009). We chose this cluster because it is representative of the high-mass end of the observed OCs. Since the total mass of the cluster system is dominated by the highmass end, the correction of biases in the mass determination are most important in that parameter regime. The cluster was set up as a $W_{0}=6 \mathrm{King}$ model with a half-mass radius of $8 \mathrm{pc}$. The extension of the cluster initially exceeds the Roche lobe initially leading to an enhanced mass loss in the first $0.5 \mathrm{Gyr}$. We used a Salpeter IMF and included mass loss by stellar evolution. The total lifetime of the cluster at a circular orbit with $R_{\mathrm{C}}=8.5 \mathrm{kpc}$ is 6.3 Gyr. For details of the evolution we refer to Just et al. (2009).

In a high-resolution simulation of a dissolving star cluster with $N=40404$ particles in the tidal field of the Milky Way the direct $N$-body code $\phi$ GRAPE $^{1}$ (Harfst et al. 2007) was used with the micro-GRAPE6 special-purpose hardware at the Astronomisches Rechen-Institut (ARI) in Heidelberg ${ }^{2}$. $\phi$ GRAPE is an acronym for Parallel Hermite Integration with GRAPE. The code is written in ANSI-C and uses a fourth-order Hermite scheme (Makino \& Aarseth 1992) for the orbit integration. It is parallelized and uses the MPI library to communicate between the processors. The force computations are executed on the fast special-purpose hardware GRAPE. The special-purpose micro-GRAPE6 hardware cards are designed especially to calculate gravitational forces in $N$-body simulations rapidly using parallelization with pipelining (see Harfst et al. 2007, and references therein).

The code $\phi$ GRAPE does not adopt regularization as the codes NBODY4 or NBODY6++ (Aarseth 1999, 2003; Spurzem 1999) do. Rather, it uses a standard Plummer type $N$-body gravitational softening. The softening length in the model used for the current work was $\epsilon=10^{-3} \mathrm{pc}$. We tested for different softening lengths $\epsilon=10^{-3}, 10^{-4}$, and $10^{-5} \mathrm{pc}$ that there are no significant differences regarding shape evolution and star-cluster mass loss.

For the simulation of a star cluster in the tidal field of the Galaxy, the $N$-body problem is solved in an analytic background potential. We use an axisymmetric three-component model, where the bulge, disc, and halo are described by PlummerKuzmin models (Miyamoto \& Nagai 1975) with the potential

$$
\Phi(R, z)=-\frac{G M}{\sqrt{R^{2}+\left(a+\sqrt{b^{2}+z^{2}}\right)^{2}}} \cdot
$$

The parameters $a, b$, and $M$ of the Milky Way model are given in Table 1 for the three components.

The top panel of Fig. 1 shows the rotation curve of the threecomponent model of the Milky Way. The parameters of the three-component model are chosen such that the rotation curve matches that of the Milky Way (Dauphole \& Colin 1995). At the

\footnotetext{
1 The present version of the code is publicly available from one of the authors FTP site: ftp://ftp . ari .uni-heidelberg.de/staff/ berczik/phi-GRAPE-cluster/code-paper/

${ }^{2}$ GRACE: http://www.ari.uni-heidelberg.de/grace
}

Table 1. The list of galaxy component parameters.

\begin{tabular}{lcrr}
\hline \hline Component & $M\left[M_{\odot}\right]$ & $a[\mathrm{kpc}]$ & $b[\mathrm{kpc}]$ \\
\hline Bulge & $1.4 \times 10^{10}$ & 0.0 & 0.3 \\
Disk & $9.0 \times 10^{10}$ & 3.3 & 0.3 \\
Halo & $7.0 \times 10^{11}$ & 0.0 & 25.0 \\
\hline
\end{tabular}

Notes. The first column gives the component, the second the mass, and the third and fourth the Plummer-Kuzmin parameters (Eq. (1)).

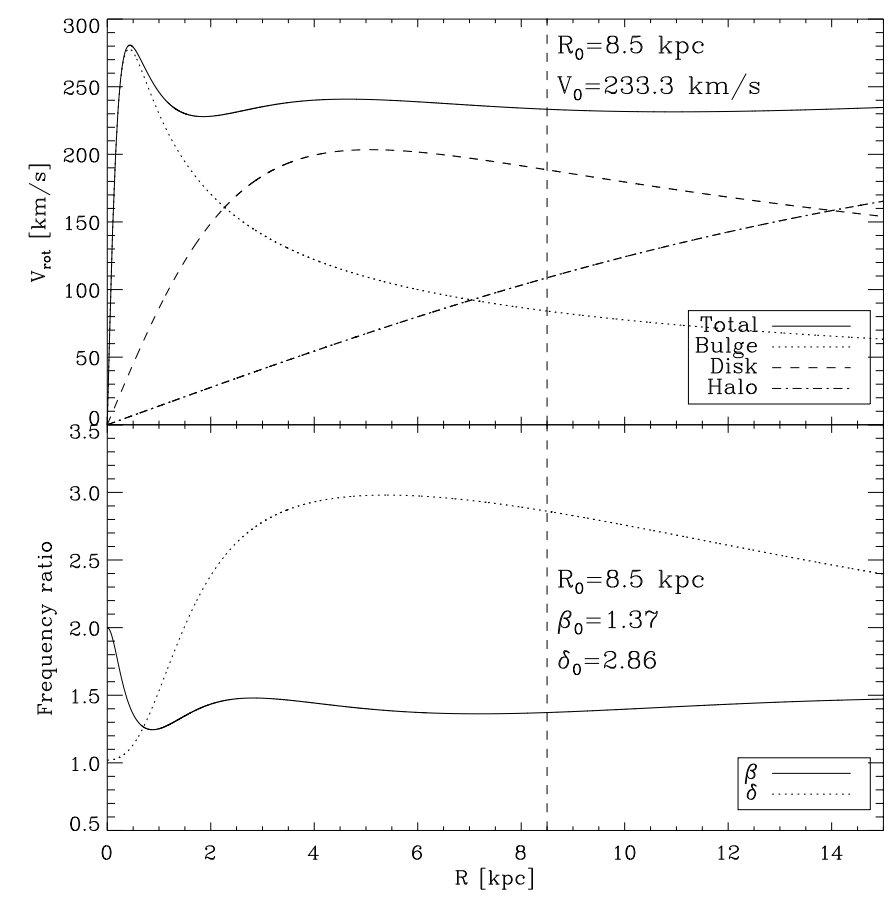

Fig. 1. Top: rotation curve (at $z=0$ ) of the three-component Plummer-Kuzmin model of the Milky Way. Bottom: epicyclic and vertical frequency parameters $\beta=\kappa / \Omega$ and $\delta=v / \Omega$ (at $z=0$ ).

solar radius $R_{0}=8.0 \mathrm{kpc}$, which was assumed in this study, the value of the circular velocity is $V_{0}=234.2 \mathrm{~km} \mathrm{~s}^{-1}$. The values of Oort's constants $A$ and $B$ are consistent with the observed values $(A, B)=(14.5 \pm 0.8,-13.0 \pm 1.1) \mathrm{km} \mathrm{s}^{-1} / \mathrm{kpc}$ derived by Piskunov et al. (2006). More generally, the dimensionless epicyclic and vertical frequency parameters are given by

$$
\begin{aligned}
& \beta^{2}=\kappa^{2} / \Omega^{2}=2\left(\frac{\mathrm{d} \ln \Omega}{\mathrm{d} \ln R}+2\right) \text { and } \\
& \delta^{2}=v^{2} / \Omega^{2}=\frac{4 \pi G \rho}{\Omega^{2}}+2-\beta^{2},
\end{aligned}
$$

where $\kappa, v$, and $\Omega$ are the epicyclic, vertical, and circular frequencies of a near-circular orbit and $\rho$ is the local Galactic density (see Oort 1965, for the derivation of $\delta^{2}$ ). The bottom panel of Fig. 1 shows the course of the epicyclic and vertical frequency parameters $\beta$ and $\delta$.

At the radius $R_{\mathrm{C}}=8.5 \mathrm{kpc}$ of the circular orbit considered in this study, we obtain $\left(\beta_{\mathrm{C}}, \delta_{\mathrm{C}}\right)=(1.37,2.86)$ and the circular velocity $V_{\mathrm{C}}=233.3 \mathrm{~km} \mathrm{~s}^{-1}$. The orbital timescale at $R_{\mathrm{C}}=8.5 \mathrm{kpc}$ is $T_{\text {orb }} \approx 224 \mathrm{Myr}$. 

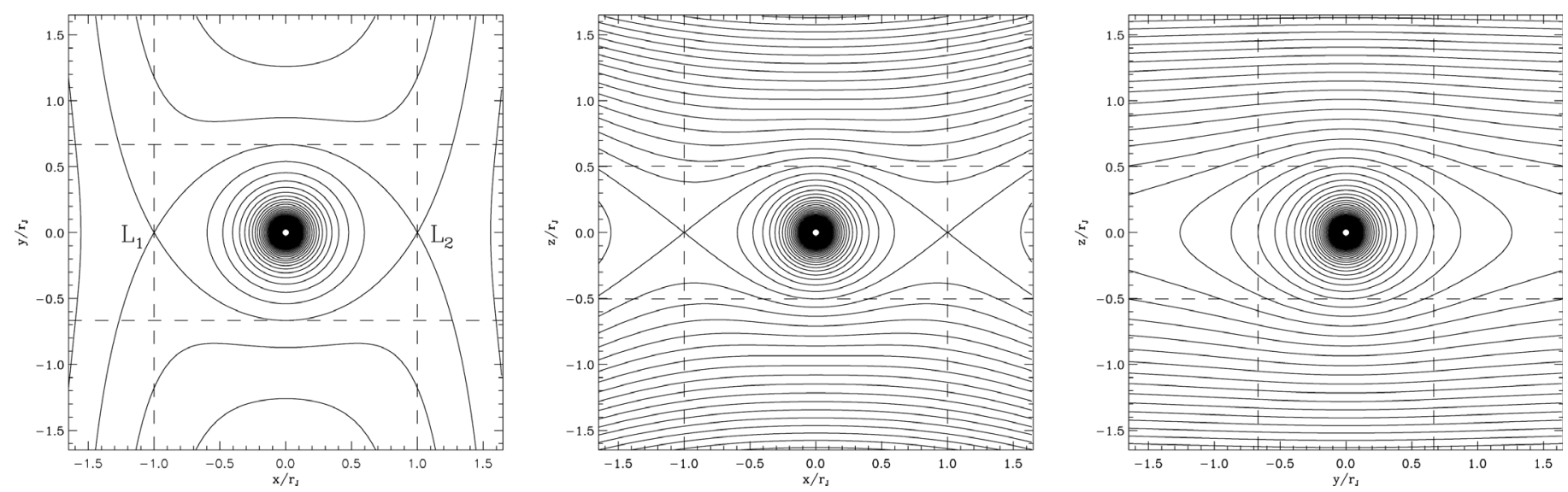

Fig. 2. Cut through the equipotential surfaces of Eq. (8) along the principal axis planes. The extents $x_{\max }$ (i.e. $r_{\mathrm{J}}$ ), $y_{\max }$ (from Eq. (11)) and $z_{\max }$ (from Eq. (15)) of the last closed equipotential surface is marked with dashed lines. We assumed a Kepler potential for the cluster.

\section{Cluster geometry}

According to King (1962), the projected density profile $\Sigma(r)$ of a star cluster can be approximated by

$\Sigma(r)=k\left\{X^{-1 / 2}-C^{-1 / 2}\right\}^{2}$ for $r \leq r_{\mathrm{t}}$

with normalization constant $k$ and

$X\left(r, r_{\mathrm{c}}\right)=1+\left(r / r_{\mathrm{c}}\right)^{2}$ and $C\left(r_{\mathrm{c}}, r_{\mathrm{t}}\right)=1+\left(r_{\mathrm{t}} / r_{\mathrm{c}}\right)^{2}$,

where $r_{\mathrm{c}}$ is the core radius and $r_{\mathrm{t}}$ is the (tidal) cutoff radius where the projected density of the model drops to zero. Integration yields the cumulative form of the King (1962) profile,

$$
\begin{aligned}
M_{\mathrm{p}}(r) & =2 \pi \int_{0}^{r} \Sigma\left(r^{\prime}\right) r^{\prime} \mathrm{d} r^{\prime} \text { for } r \leq r_{\mathrm{t}} \\
& =\pi r_{\mathrm{c}}^{2} k\left\{\ln (X)-4 \frac{X^{1 / 2}-1}{C^{1 / 2}}+\frac{X-1}{C}\right\}
\end{aligned}
$$

where $M_{\mathrm{p}}(r)$ is the projected cumulative mass of the model, i.e. the mass in projection on the sky within a circle of radius $r$. For $r>r_{\mathrm{t}}$, we force the integrated profile (Eq. (6)) to the finite value

$M_{\mathrm{p}}\left(r_{\mathrm{t}}\right)=\pi r_{\mathrm{c}}^{2} k\left\{\ln (C)-3+\frac{4}{C^{1 / 2}}-\frac{1}{C}\right\}$.

We do not use the identification of $M_{\mathrm{p}}\left(r_{\mathrm{t}}\right)$ with the cluster mass, since in practice the equations are applied to star counts and the effective mass-of-light ratio enters the normalization constant $k$.

Already King (1961) remarks that the tidal forces from the galaxy distort only the outer regions of a star cluster. We quantify these deviations from spherical symmetry caused by the tidal field in this section. We employ a coordinate system $(x, y, z)$ of "principal axes of the star cluster". Its origin is the cluster center. The $x$-axis points away from the Galactic center, the $y$-axis points in the direction of the Galactic rotation and the $z$-axis is directed towards the Galactic north pole. Figure 2 shows a "principal axis plane cut" through the equipotential surfaces of the effective potential around a star cluster on a circular orbit in the tidal field of the Milky Way. To second order, the effective potential is given by

$\Phi_{\mathrm{eff}}=\Phi_{\mathrm{eff}, 0}-\frac{G M_{\mathrm{cl}}}{\sqrt{x^{2}+y^{2}+z^{2}}}+\frac{1}{2}\left(\beta^{2}-4\right) \Omega^{2} x^{2}+\frac{1}{2} \delta^{2} \Omega^{2} z^{2}$.

For the cluster, we assumed a Kepler potential, which is a very good approximation in the outer parts (Just et al. 2009). The unit in Fig. 2 is the Jacobi radius $r_{\mathrm{J}}$. The Jacobi radius is defined as the distance from the cluster center to the Lagrange points $L_{1}$ and $L_{2}$. It is given by

$r_{\mathrm{J}}=\left[\frac{G M_{\mathrm{cl}}}{\left(4-\beta^{2}\right) \Omega^{2}}\right]^{1 / 3}$

(see King 1962). The value of the effective potential on the critical equipotential surface, which connects $L_{1}$ and $L_{2}$, can be easily calculated from Eqs. (8) and (9). It is given by

$\Phi_{\text {eff,crit }}=\Phi_{\mathrm{eff}, 0}-\frac{3}{2} \frac{G M_{\mathrm{cl}}}{r_{\mathrm{J}}}$.

Assuming that $x_{\max }=r_{\mathrm{J}}$, the radius in the $y$-direction of the last closed (critical) equipotential surface follows from Eqs. (8) and (10),

$y_{\max }=\frac{2}{3} r_{\mathrm{J}}$.

For the radius in $z$-direction, we have to solve a cubic equation

$-\frac{K}{z_{\max }}+\frac{1}{2} L^{2} z_{\max }^{2}=-\frac{3}{2} \frac{K}{M}$

with the constants

$K=G M_{\mathrm{cl}}, \quad L=\delta \Omega, \quad M=r_{\mathrm{J}}$

The only real solution is given by

$z_{\max }=\frac{\left\{K\left[K+2 L\left(L M^{3}+\sqrt{K M^{3}+L^{2} M^{6}}\right)\right]\right\}^{1 / 3}-K^{2 / 3}}{L\left(L M^{3}+\sqrt{K M^{3}+L^{2} M^{6}}\right)^{1 / 3}}$.

For $(\beta, \delta)=\left(\beta_{\mathrm{C}}, \delta_{\mathrm{C}}\right)=(1.37,2.86)$, we obtain

$z_{\max } \approx 0.503 r_{\mathrm{J}}$

(e.g. Wielen 1974). More generally, the ratios of the principal axes $y_{\max }$ to $x_{\max }$ and $z_{\max }$ to $x_{\max }$ of all closed equipotential surfaces are shown in Fig. 3 as a function of the parameter $\gamma=x / r_{\mathrm{J}}$. At the center of the cluster we have $\gamma=0$, while $\gamma=1$ corresponds to the critical equipotential surface through $x=r_{\mathrm{J}}$.

Figure 4 shows the logarithmically color-coded surface density $\Sigma$ of projections of the simulated $N$-body model of the star cluster onto its principal axis planes at time $T_{4}=1.31 \mathrm{Gyr}$. The surface density $\Sigma$ has been calculated from the $N$-body snapshot 

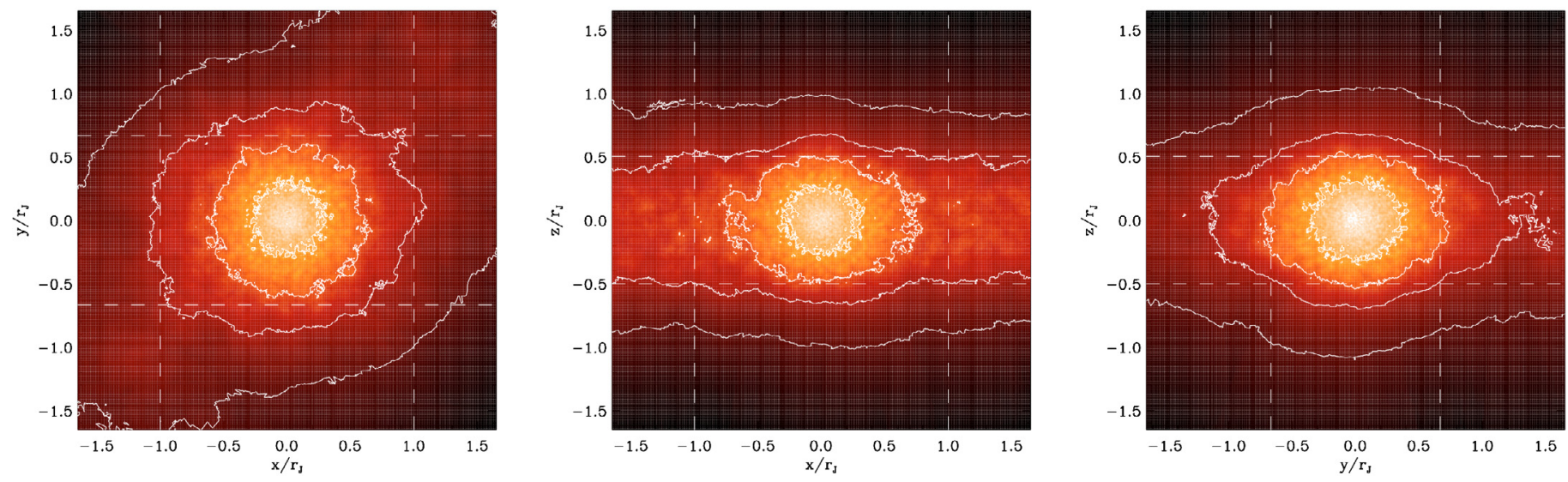

Fig. 4. Surface density of projections onto the principal axis planes of the cluster at $T_{4}=1.31 \mathrm{Gyr}$. The dashed lines show the theoretical values of $x_{\max } / r_{\mathrm{J}}, y_{\max } / r_{\mathrm{J}}$, and $z_{\max } / r_{\mathrm{J}}$. The contours correspond to $\Delta \log \Sigma \approx 2$ dex.

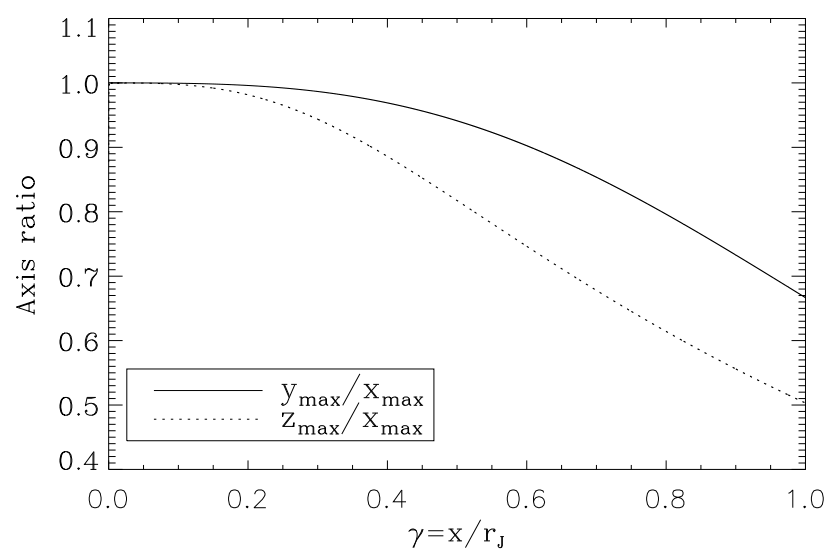

Fig. 3. Principal axis ratios of the equipotential surfaces around the cluster as a function of the parameter $\gamma=x / r_{\mathrm{J}}$. At the center of the cluster, we have $\gamma=0$ while $\gamma=1$ (dashed line) corresponds to the critical equipotential surface through $x=r_{\mathrm{J}}$. We assumed $(\beta, \delta)=(1.37,2.86)$ and a Kepler potential for the cluster.

file with the method of Casertano \& Hut (1985) (their Eq. (II.6) with $j=20$ ). The contours correspond to $\Delta \log \Sigma \approx 2$ dex. The extent of the last closed (critical) equipotential surface is marked with dashed lines. The contours of constant surface density roughly follow the equipotential surfaces from Fig. 2.

\section{Jacobi radius of the model}

We determined the Jacobi radius of our simulated $N$-body model iteratively from Eq. (9). The gravitational constant $G$ and the quantities $\beta$ and $\Omega$ are known but $M_{\mathrm{cl}}$, the cluster mass within $r=r_{\mathrm{J}}$ is unknown since $r_{\mathrm{J}}$ is unknown. Our iteration is given by

$r_{n+1}=\left[\frac{G M_{\mathrm{enc}}\left(r_{n}\right)}{\left(4-\beta^{2}\right) \Omega^{2}}\right]^{1 / 3}$

where $M_{\mathrm{enc}}(r)$ is the enclosed mass of cluster stars within radius $r$ around the cluster center. Starting with $r_{0}=\infty$, the iteration theoretically converges towards $r_{\infty}=r_{\mathrm{J}}$. In practice, a few iterations are sufficient to determine $r_{\mathrm{J}}$ accurately. We note that this simple method does not rely on numerical fits of the effective potential to the envelope of the spatial distribution of Jacobi energies of the cluster stars. It can easily be applied to an $N$-body snapshot file that contains masses and positions of the individual particles at a certain time.

\section{Projection and fitting}

We calculate the projection at the sky in Galactic coordinates $(l, b)$ and ignore for simplicity the perspective effects. Strictly speaking the projection of the cluster model is consistent only in the midplane $b=0^{\circ}$ corresponding to the circular orbit in the Galactic plane. The rotation angle $\alpha$ is related to the Galactic longitude $l$ by the law of sines

$\sin \alpha=-\sin l \frac{R_{0}}{R_{\mathrm{C}}} \quad R_{\mathrm{C}}>R_{0}$

where $R_{0}=8 \mathrm{kpc}$ at the solar circle and $R_{\mathrm{C}}=8.5 \mathrm{kpc}$ at the cluster orbit. At the Galactic coordinates $(l, b)=(0,0)$ and $(l, b)=\left(180^{\circ}, 0\right)$, the "system $(x, y, z)$ of principal axes of the cluster" with origin at the cluster center is not rotated. We note that at $l=90^{\circ}$ and $l=270^{\circ}$ we have the maximum rotation angle $\alpha=-70^{\circ} .25$ and $\alpha=70^{\circ} .25$, respectively.

A sketch of the projection of the simulated $N$-body model of the star cluster onto the plane of the sky is illustrated in Fig. 5. Four orbital positions of the dissolving star cluster with its tidal tails are marked in the sketch.

To demonstrate the effect of different projections also in Galactic latitude, we add the cases of a certain height of the cluster orbit above and below the plane of the solar circle (dotted lines in Fig. 5). The effect of the corresponding vertical oscillation of the cluster orbit on the intrinsic structure of the cluster and of a variation in $\delta$ in Eq. (3) are neglected in this study.

For all positions of the cluster on its orbit, we rotate the cluster around the $z$ axis and then around the $y^{\prime}$ axis by the ordered pair of angles $(\alpha,-b)$ to simulate the perspective of the cluster for an observer on earth. After the projection, we determine the polar symmetric profile of the projected cumulative mass $M_{\mathrm{p}}(r)$ from our $N$-body data file by summations over radius and polar angle and apply a fit with Eq. (6). For the fitting, we used 
the MPFIT package in IDL (Markwardt 2009; Moré 1978, for the Levenberg-Marquardt algorithm).

\section{Results}

We now discuss projections according to Eq. (17) in detail. Figure 6 shows examples of fits (upper panels) with the corresponding projections (lower panels). The resulting parameter ratio $r_{\mathrm{t}} / r_{\mathrm{J}}$ is given in the upper panels and the $(l, b)$ coordinates in the corresponding lower panels. In the upper panels, the solid (black) line represents the data and dotted (blue) line the fit. The dashed (red) lines indicate $r_{\mathrm{c}}$ (left dashed line) and $r_{\mathrm{t}}$ (right dashed line) derived in the fit with Eq. (6). In the lower panels, the dashed (red) line indicates $r_{\mathrm{J}}$.

We derived the ratio $r_{\mathrm{t}} / r_{\mathrm{J}}$ for all projection directions in $(l, b)$ at four different evolution times of the cluster. The projections are performed in steps of 4 degrees from $b=-90^{\circ}$ to $b=+90^{\circ}$ and $l=0$ to $l=360^{\circ}$. The full $N$-body snapshot file with $N=40404$ particles has been used for the projection and fitting procedure as described in Sect. 5. Figure 7 shows parameter surfaces of $r_{\mathrm{t}} / r_{\mathrm{J}}$ as a function of Galactic coordinates of the cluster center at times $T_{1}=0.62 \mathrm{Gyr}$ (top left), $T_{2}=0.84 \mathrm{Gyr}$ (top right), $T_{3}=1.06 \mathrm{Gyr}$ (bottom left) and $T_{4}=1.31 \mathrm{Gyr}$ (bottom right). A squeezed Hammer-Aitoff projection of Galactic coordinates has been used (see Appendix A). The total number of fits for each plot in Fig. 7 is $N_{\text {fits }}=4186$. We note that in the plot for $T_{1}$, a peak around $(l, b) \approx\left(270^{\circ}, 0\right)$ (see also Fig. A.1 in Appendix A) is not resolved (white colored area). Here $r_{\mathrm{t}} / r_{\mathrm{J}}$ reaches a factor of 1.52 .

It can be seen that the cluster masses are typically overestimated. The strongest bias to high masses at time $T_{1}$ (including the peak) shows that the unbound stars stay for a long time close to the cluster and contribute to the outer density profile in the fitting procedure. This bias depends on the initial conditions. At later times $T_{3}, T_{4}$, the cluster mass distribution becomes stable and the bias is independent of the age of the cluster.

Figure 8 shows a histogram of the fraction on the sky per bin in $M_{\mathrm{t}} / M_{\mathrm{cl}}=\left(r_{\mathrm{t}} / r_{\mathrm{J}}\right)^{3}$ for the parameter surface in Fig. 7 corresponding to time $T_{4}$, where the "tidal" masses $M_{\mathrm{t}}$ and $M_{\mathrm{cl}}$ are calculated with Eq. (9) from $r_{\mathrm{t}}$ and $r_{\mathrm{J}}$, respectively. The solid line shows the distribution for projections in the range $-20^{\circ}<b<+20^{\circ}$. The most frequent overestimation of the mass is $M_{\mathrm{t}} / M_{\mathrm{cl}}=1.18$. The dashed line shows the distribution for projections in the range $-40^{\circ}<b<+40^{\circ}$. The most frequent ratio is the same as for the solid line. However, the ratios extend up to $M_{\mathrm{t}} / M_{\mathrm{cl}}=1.7$. The dotted line shows the distribution for all projections. The most frequent ratio is the same as for the other two lines, but the ratios extend up to $M_{\mathrm{t}} / M_{\mathrm{cl}} \approx 2.1$.

Figure 9 shows the mean mass $\left\langle M_{\mathrm{t}} / M_{\mathrm{cl}}\right\rangle$ as a function of the Galactic latitude $b$, where $M_{\mathrm{t}}$ is the mass of cluster stars within radius $r_{\mathrm{t}}$ derived from the fitting procedure. Shown are the curves corresponding to times $T_{1}-T_{4}$. All curves show a local minimum located roughly at $b=0^{\circ}$. For time $T_{1}$, the mean overestimation of the mass reaches 2.5 in the direction of the Galactic poles.

Only a small fraction of cluster stars are usually identified as members leading to an increased statistical uncertainty in the fitting procedure. The uppermost plot in Fig. 10 shows the parameter surface derived for the 400 most massive (i.e. the brightest) stars in the simulation at time $T_{4}$ (data courtesy of Beuria). The small values of $r_{\mathrm{t}} / r_{\mathrm{J}}$ are caused by mass segregation. The stronger asymmetries with respect to $b=0^{\circ}$ compared to the plots in Fig. 7 are due to a slightly asymmetric distribution of the 400 mass-segregated stars in position space.

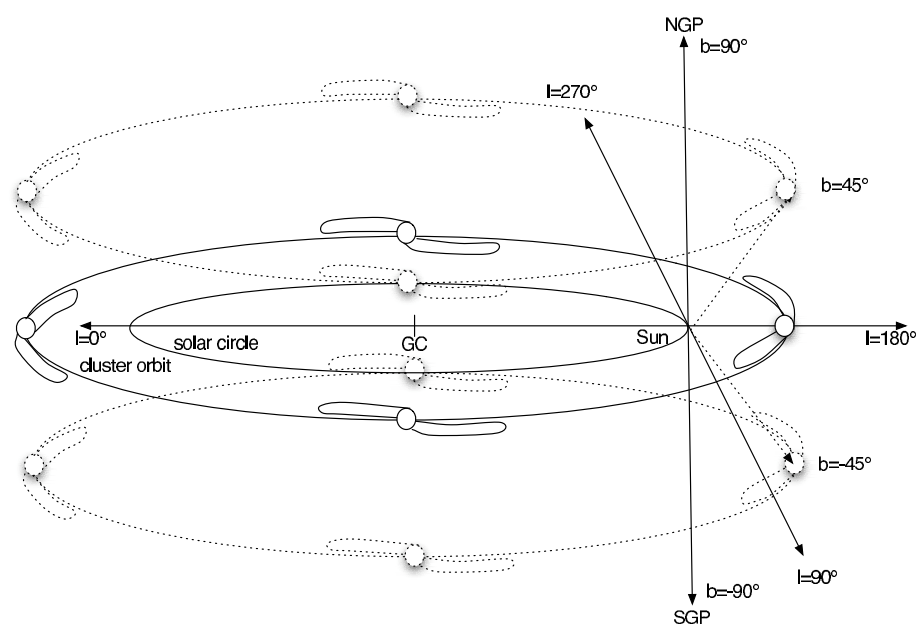

Fig. 5. Sketch of the Galactic coordinate system in which the projection is done. The Galactocentric radius of the Sun is assumed to be $R_{0}=$ $8.0 \mathrm{kpc}$, while the cluster orbits at $R_{\mathrm{C}}=8.5 \mathrm{kpc}$ on a circular orbit. The abbreviations denote the Galactic north (NGP) and south (SGP) Pole and the Galactic center (GC).

In order to measure the statistical scatter in $r_{\mathrm{t}}$, we applied a bootstrap analysis. We divided the $N$-body snapshot file for time $T_{4}$ with $N=40404$ particles into 100 small-number samples of $N_{\text {sample }}=400$ particles each (the remaining particles were dropped out of the analysis). For each of these small-number samples we applied the procedure described above. The resulting total number of projections and fits was therefore $N_{\text {fits }}=$ 418 600. The middle and bottom plots in Fig. 10 show the results of the bootstrap analysis. The middle plot of Fig. 10 shows the mean value of $r_{\mathrm{t}} / r_{\mathrm{J}}$ averaged over the 100 samples. The bottom plot shows the relative standard deviation $\Delta r_{\mathrm{t}} / r_{\mathrm{t}}$ evaluated by averaging over the 100 samples. The uncertainty in a single determination of $r_{\mathrm{t}}$ is between $10 \%$ and $20 \%$. The highest uncertainty is expected for $r_{\mathrm{t}}$-determinations in the vicinity of the peak around $(l, b) \approx\left(270^{\circ}, 0\right)$.

A comparison with the uppermost plot in Fig. 10 shows that the derived limiting radii for the sample of the most massive stars are systematically lower because of mass segregation. The differences are typically on a $1-2 \sigma$ level.

\section{Conclusions}

In this paper, we have confirmed that star cluster masses are typically overestimated if the method of Piskunov et al. (2007) is applied to a complete sample. Moreover, we have quantified the methodological error in our analysis.

Figures 7 and 10 show that at certain Galactic coordinates the King (1962) profile fits are particularly biased. A high bias is predicted for $(l, b) \approx\left(270^{\circ}, 0\right)$ (see Figs. 7,10 , and A.1). The corresponding rotation angle of the cluster is $\alpha=70^{\circ}$, where the projection is parallel to the inner end of the tidal arms (see lower left plots in Fig. 6). For $(l, b)=\left(90^{\circ}, 0\right)$, there is no corresponding peak in the parameter surface (most clearly visible for $T_{1}$ ) because of the asymmetry between the leading and trailing tidal tails (see the sketch in Fig. 5). For Galactic latitudes beyond $b \approx \pm 40^{\circ}$ (cf. Fig. A.1), the bias becomes large but only a few OCs occupy this regime.

For the parameter surfaces in Fig. 7, the masses are biased within the ranges $\left[1.3 M_{\mathrm{cl}}, 3.5 M_{\mathrm{cl}}\right]\left(\right.$ at $\left.T_{1}\right),\left[1.1 M_{\mathrm{cl}}, 2.3 M_{\mathrm{cl}}\right]$ (at $\left.T_{2}\right),\left[1.0 M_{\mathrm{cl}}, 2.0 M_{\mathrm{cl}}\right]\left(\right.$ at $\left.T_{3}\right)$ and $\left[1.0 M_{\mathrm{cl}}, 2.1 M_{\mathrm{cl}}\right]\left(\right.$ at $\left.T_{4}\right)$ depending on the evolutionary state of the star cluster in the tidal 
A\&A 524, A62 (2010)
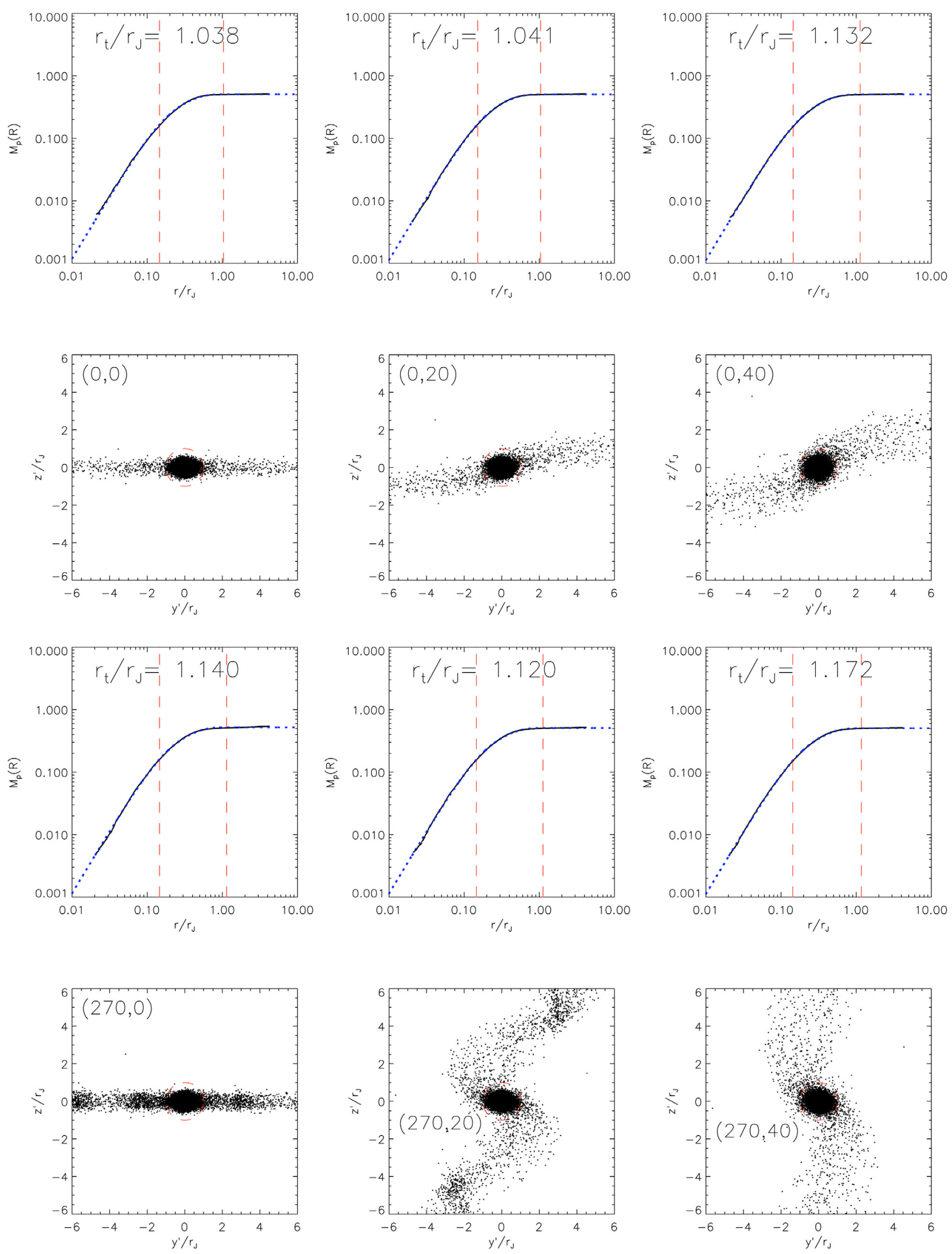

Fig. 6. Examples of fits (upper panels) with the corresponding projections (lower panels) at time $T_{4}=1.31$ Gyr. The resulting parameter ratio $r_{\mathrm{t}} / r_{\mathrm{J}}$ is given in the upper panels and the $(l, b)$ coordinates in the corresponding lower panels. In the upper panels, the solid (black) line represents the data and dotted (blue) line the fit. The dashed (red) lines mark $r_{\mathrm{c}}$ (left dashed line) and $r_{\mathrm{t}}$ (right dashed line) from the fit with Eq. (6). In the lower panels, the dashed (red) line marks $r_{\mathrm{J}}$. 
A. Ernst et al.: Calibration of radii and masses of OCs
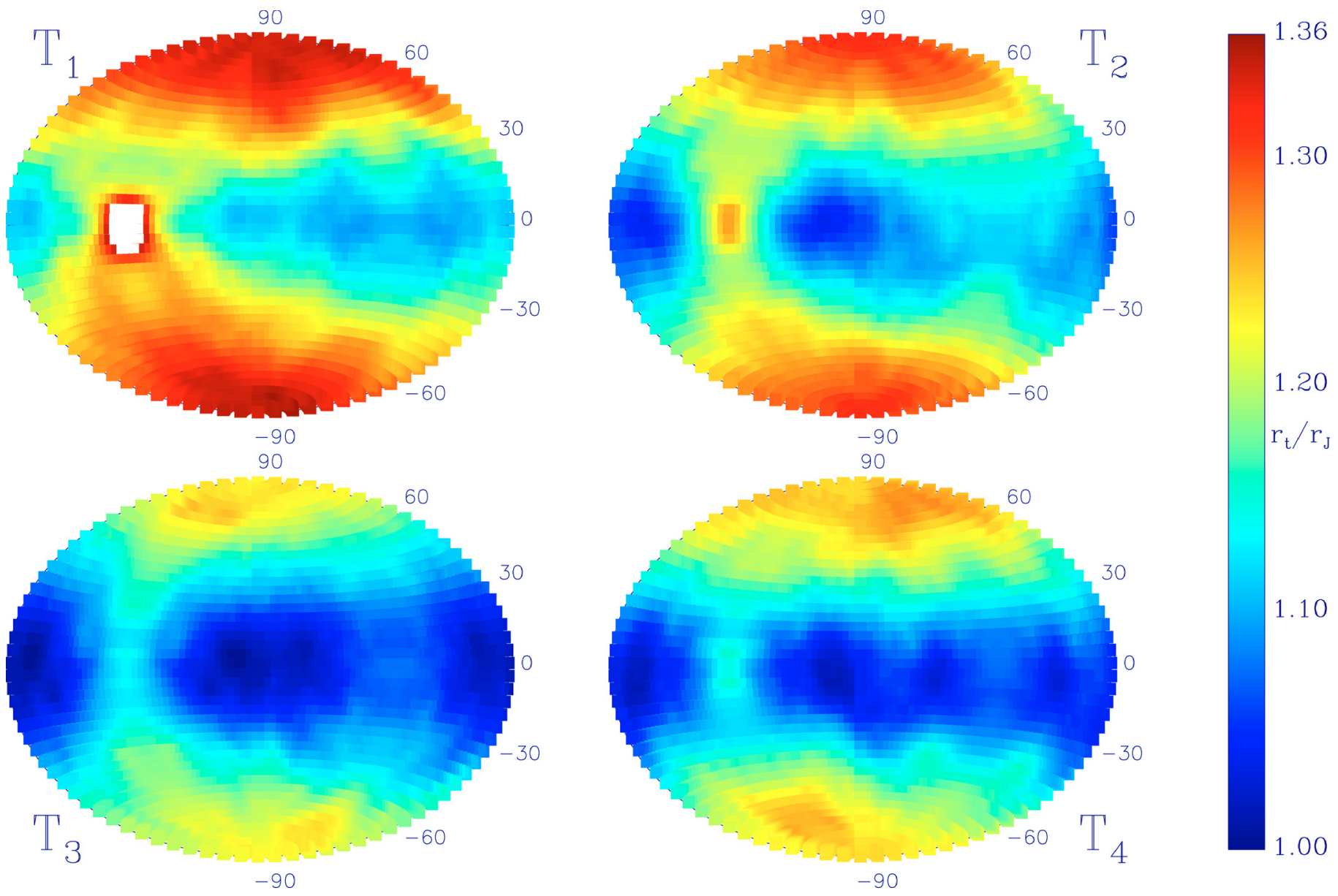

Fig. 7. Parameter surfaces of $r_{\mathrm{t}} / r_{\mathrm{J}}$ as a function of Galactic coordinates for a fit of King (1962) models to projections on the sky of a simulated model at different positions on its theoretical orbit. We used a squeezed Hammer-Aitoff projection. The color denotes the value of $r_{\mathrm{t}} / r_{\mathrm{J}}$ on a linear scale. The plots in the top row correspond to $T_{1}=0.62 \mathrm{Gyr}$ (top left) and $T_{2}=0.84 \mathrm{Gyr}$ (top right). The plots in the bottom row correspond to $T_{3}=1.06 \mathrm{Gyr}$ (bottom left) and $T_{4}=1.31 \mathrm{Gyr}$ (bottom right).

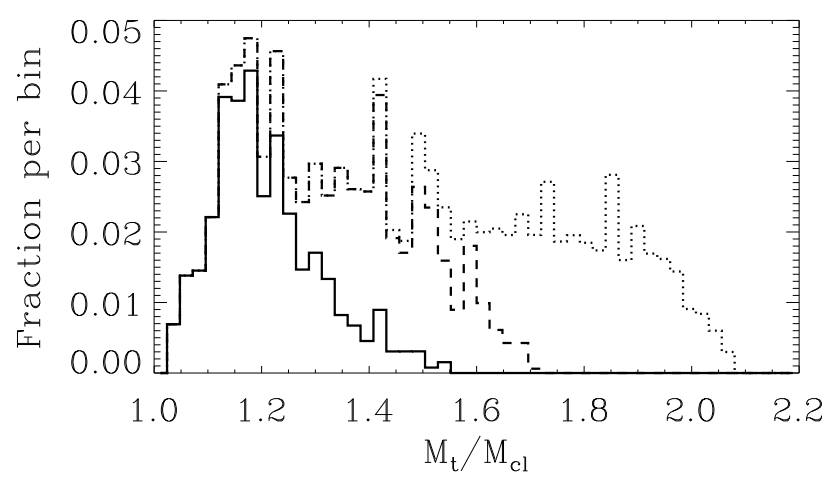

Fig. 8. Histogram of the fraction on the sky per bin in $M_{\mathrm{t}} / M_{\mathrm{cl}}$ corresponding to the $T_{4}=1.31 \mathrm{Gyr}$ parameter surface from Fig. 7. $M_{\mathrm{t}}$ and $M_{\mathrm{cl}}$ are the "tidal masses" calculated with Eq. (9) from $r_{\mathrm{t}}$ and $r_{\mathrm{J}}$, respectively.

field of the Galaxy. The bias depends strongly on the projection angles, which transform differently into Galactic coordinates for different orbital radii of the OC.

Furthermore, we have demonstrated using a bootstrap analysis that the relative error in a single determination of the limiting

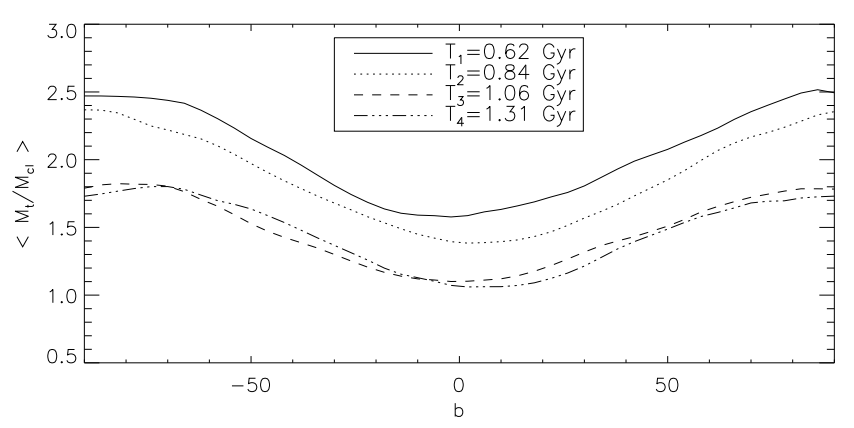

Fig. 9. Mean mass $\left\langle M_{\mathrm{t}} / M_{\mathrm{cl}}\right\rangle$ as a function of the Galactic latitude $b . M_{\mathrm{t}}$ and $M_{\mathrm{cl}}$ are the "tidal masses" calculated with Eq. (9) from $r_{\mathrm{t}}$ and $r_{\mathrm{J}}$, respectively.

radius $r_{\mathrm{t}}$ is between $10 \%$ and $20 \%$ (at time $T_{4}$ ) corresponding to an uncertainty in the mass of $\approx 50 \%$ for samples of $N_{\text {sample }}=$ 400 particles (which are typical of rich OCs).

We have shown that the mass segregation of the brightest stars in a cluster can alter the $r_{\mathrm{t}} / r_{\mathrm{J}}$ factor significantly, which is an important result for the data analysis of observations. The mass segregation results in a concentrated core that causes an 

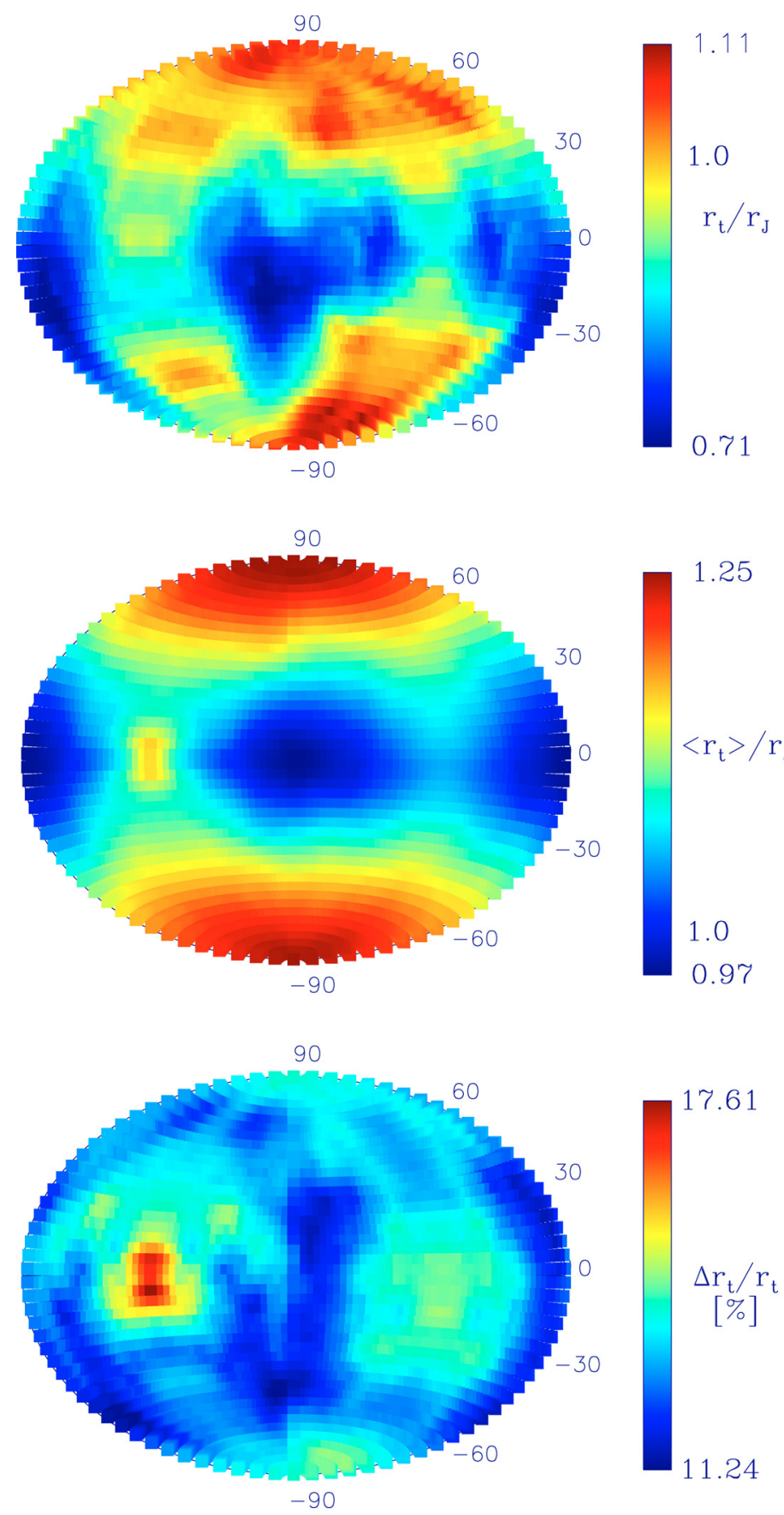

Fig. 10. Parameter surfaces of $r_{\mathrm{t}} / r_{\mathrm{J}}$ and $\Delta r_{\mathrm{t}} / r_{\mathrm{t}}$ as a function of Galactic coordinates for a fit of King (1962) models to to projections on the sky of a simulated model at different positions on its theoretical orbit. We used a squeezed Hammer-Aitoff projection. The time is $T_{4}=1.31 \mathrm{Gyr}$. The color denotes the value of $r_{\mathrm{t}} / r_{\mathrm{J}}$ (and $\Delta r_{\mathrm{t}} / r_{\mathrm{t}}$, bottom plot) on a linear scale. The upper plots shows the parameter surface for the 400 brightest stars in the simulated cluster (data courtesy of Beuria). The middle and bottom plots show the result of the bootstrap analysis (for explanations see the text). The middle plot shows the mean value of $r_{\mathrm{t}} / r_{\mathrm{J}}$ averaged over 100 small-number samples. The bottom plot shows the corresponding relative standard deviation $\Delta r_{\mathrm{t}} / r_{\mathrm{t}}$.

underestimation of the tidal radius. For a younger cluster age, one would expect a smaller amount of mass segregation.

To apply a quantitative correction for the bias in the cluster mass determination by identifying $r_{\mathrm{t}}$ with $r_{\mathrm{J}}$ an extensive parameter study of cluster parameters is necessary. The influence of mass-segregation on selection effects caused by the brightness

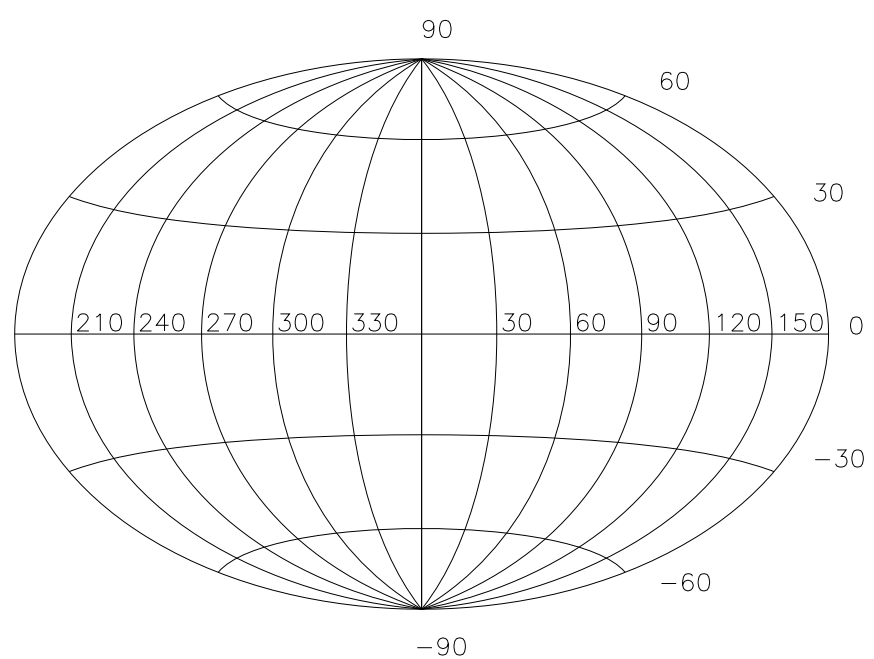

Fig. A.1. Squeezed Hammer-Aitoff projection. The Galactic latitude runs from $b=-90^{\circ}$ to $b=+90^{\circ}$ and the longitude from $l=0$ to $l=360^{\circ}$.

limit of the observations should be considered by such a study, because stellar evolution is taken into account. For young clusters, the bias factor can be particularly sensitive to the initial conditions. The final goal is to find an agreement between the OC mass determinations by the different methods. This would allow an interesting insight into the OC properties such as the IMF, mass-to-light ratio, and mass segregation.

Acknowledgements. The authors thank J. Beuria for his help with the HammerAitoff projections and the provision of the data for the uppermost plot in Fig. 10. P.B. and M.I.P. acknowledge the special support by the Ukrainian National Academy of Sciences under the Main Astronomical Observatory GRAPE/GRID computing cluster project. P.B. acknowledges the support from the Volkswagen Foundation GRACE Project No. I80 041-043. M.I.P. acknowledges support by the University of Vienna through the frame of the Initiative Kolleg (IK) "The Cosmic Matter Circuit" I033-N and computing time on the Grape Cluster of the University of Vienna.

\section{Appendix A: Squeezed Hammer-Aitoff projection}

The squeezed Hammer-Aitoff projection is given by

$$
\begin{aligned}
& x=2 f \frac{\cos (b) \sin (l / 2)}{\sqrt{1+\cos (b) \cos (l / 2)}}, \\
& y=\frac{2}{f} \frac{\sin (b)}{\sqrt{1+\cos (b) \cos (l / 2)}} .
\end{aligned}
$$

It represents the standard Hammer-Aitoff equal-area projection, where we have also introduced a free squeezing factor $f$. The squeezing leaves the area element $\mathrm{d} A=\mathrm{d} x \mathrm{~d} y=\sqrt{\operatorname{det} g} \mathrm{~d} b \mathrm{~d} l=$ $\cos (b) \mathrm{d} b \mathrm{~d} l$ invariant, where $g$ is the first fundamental form calculated from Eqs. (A.1) and (A.2). The ratio of diameters of the elliptic projection area is given by $d_{x} / d_{y}=f^{2}$. For the standard Hammer-Aitoff projection, we have $f=\sqrt{2}$. For a projection onto a circular area one can set $f=1$. The inverse projection is given by

$$
\begin{aligned}
& z=\sqrt{2-\left(\frac{x}{2 f}\right)^{2}-\left(\frac{f y}{2}\right)^{2}}, \\
& b=\arcsin \left(\frac{f}{2} z y\right),
\end{aligned}
$$


A. Ernst et al.: Calibration of radii and masses of OCs

$l=2 \arctan \left(\frac{1}{2 f} \frac{z x}{z^{2}-1}\right)$,

where we have introduced the auxiliary variable $z$.

We assumed that $f=1.2$ for Figs. 7 and 10. The resulting coordinate system (which is hidden in Figs. 7 and 10) can be seen in Fig. A.1. We modified IDL routines by Landsman to incorporate the free squeezing factor.

\section{References}

Aarseth, S. J. 1999, PASP, 111, 1333

Aarseth, S. J. 2003, Gravitational N-body simulations - Tools and Algorithms (Cambridge Univ. Press)

Casertano, S., \& Hut, P. 1985, ApJ, 298, 80

Dauphole, B., \& Colin, J. 1995, A\&A, 300, 117

Harfst, S., Gualandris, A., Merritt, D., et al. 2007, New Astron., 12, 357

Just, A., Berczik, P., Petrov, M. I., \& Ernst, A. 2009, MNRAS, 392, 969

Kharchenko, N. V., Berczik, P., Petrov, M. I., et al. 2009, A\&A, 495, 807

King, I. 1961, AJ, 66, 68
King, I. 1962, AJ, 67, 471

Makino, J., \& Aarseth, S. J. 1992, PASJ, 44, 141

Markwardt, C. B. 2009, in proc. Astronomical Data Analysis Software and Systems XVIII, Quebec, Canada, ed. D. Bohlender, P. Dowler, \& D. Durand, San Francisco, ASP Conf. Ser., 411, 251

Miller, G. E., \& Scalo, J. M. 1978, PASP, 90, 506

Miyamoto, M., \& Nagai, R. 1975, PASJ, 27, 533

Moré, J. 1978, in Numerical Analysis, ed. G. A. Watson (Berlin: Springer Verlag), 630, 105

Oort, J. H. 1965, in Galactic Structure, ed. A. Blaauw, \& M. Schmidt (Chicago, IL: Univ. Chicago Press), 455

Piskunov, A. E., Schilbach, E., Kharchenko, N. V., Röser, S., \& Scholz, R.-D. 2007, A\&A, 468, 151

Piskunov, A. E., Schilbach, E., Kharchenko, N. V., Röser, S., \& Scholz, R.-D. 2008a, A\&A, 477, 165

Piskunov, A. E., Kharchenko, N. V., Schilbach, E., et al. 2008b, A\&A, 487, 557 Roeser, S., Kharchenko, N. V., Piskunov, A. E., et al. 2010, Astron. Nachr., 331, 519

Spurzem, R. J. 1999, Comp. Applied Maths., 109, 407

Wielen, R. 1971, A\&A, 13, 309

Wielen, R. 1974, in Proceedings of the 1st European Astronomical Meeting, Stars and the Milky Way System, ed. L. N. Mavridis (Berlin: Springer), 2, 326 